\title{
Genomic investigation of Danish Staphylococcus aureus isolates from bulk tank milk and dairy cows with clinical mastitis
}

Ronco, Troels; Klaas, Ilka C.; Stegger, Marc; Svennesen, Line; Astrup, Lærke Boye; Farre, Michael; Pedersen, Karl

Published in:

Veterinary Microbiology

Link to article, DOI:

10.1016/j.vetmic.2018.01.003

Publication date:

2018

Document Version

Peer reviewed version

Link back to DTU Orbit

Citation (APA):

Ronco, T., Klaas, I. C., Stegger, M., Svennesen, L., Astrup, L. B., Farre, M., \& Pedersen, K. (2018). Genomic investigation of Danish Staphylococcus aureus isolates from bulk tank milk and dairy cows with clinical mastitis. Veterinary Microbiology, 215, 35-42. https://doi.org/10.1016/j.vetmic.2018.01.003

\section{General rights}

Copyright and moral rights for the publications made accessible in the public portal are retained by the authors and/or other copyright owners and it is a condition of accessing publications that users recognise and abide by the legal requirements associated with these rights.

- Users may download and print one copy of any publication from the public portal for the purpose of private study or research.

- You may not further distribute the material or use it for any profit-making activity or commercial gain

- You may freely distribute the URL identifying the publication in the public portal 


\section{Accepted Manuscript}

Title: Genomic investigation of Danish Staphylococcus aureus isolates from bulk tank milk and dairy cows with clinical mastitis

Authors: Troels Ronco, Ilka C. Klaas, Marc Stegger, Line Svennesen, Lærke B. Astrup, Michael Farre, Karl Pedersen

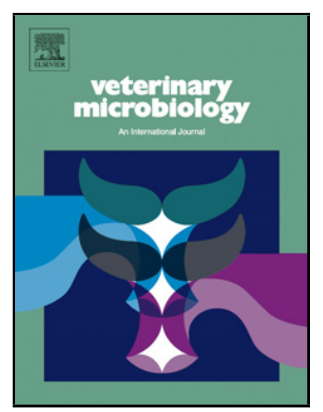

PII: S0378-1135(17)31309-3

DOI: https://doi.org/10.1016/j.vetmic.2018.01.003

Reference: VETMIC 7845

To appear in: $\quad$ VETMIC

Received date: $\quad 8-11-2017$

Revised date: $\quad 8-1-2018$

Accepted date: $\quad$ 9-1-2018

Please cite this article as: Ronco, Troels, Klaas, Ilka C., Stegger, Marc, Svennesen, Line, Astrup, Lærke B., Farre, Michael, Pedersen, Karl, Genomic investigation of Danish Staphylococcus aureus isolates from bulk tank milk and dairy cows with clinical mastitis.Veterinary Microbiology https://doi.org/10.1016/j.vetmic.2018.01.003

This is a PDF file of an unedited manuscript that has been accepted for publication. As a service to our customers we are providing this early version of the manuscript. The manuscript will undergo copyediting, typesetting, and review of the resulting proof before it is published in its final form. Please note that during the production process errors may be discovered which could affect the content, and all legal disclaimers that apply to the journal pertain. 
Genomic investigation of Danish Staphylococcus aureus isolates from bulk tank milk and dairy cows with clinical mastitis

Short title: Analysis of S. aureus isolates from dairy Farms

Troels Ronco ${ }^{\mathrm{a}^{*}}$, Ilka C. Klaas ${ }^{\mathrm{b}}$, Marc Stegger ${ }^{\mathrm{c}}$, Line Svennesen ${ }^{\mathrm{b}}$, Lærke B. Astrup ${ }^{\mathrm{a}}$, Michael Farre ${ }^{\mathrm{d}}$, Karl Pedersen $^{\mathrm{a}}$

${ }^{a}$ National Veterinary Institute, Technical University of Denmark, Kemitorvet build. 204, 2800 Kgs. Lyngby, Denmark, 'bection for Production, Nutrition and Health, University of Copenhagen, Grønnegårdsvej 2, 1870 Fdr. C, Denmark, ' Department of Bacteria, Parasites and Fungi, Statens Serum Institut, Artillerivej 5, 2300 Copenhagen S, Denmark, ${ }^{\mathrm{d}}$ SEGES Livestock Innovation, Agro Food Park 15, 8200 Aarhus N, Denmark

Corresponding author: Troels Ronco, troro@ vet.dtu.dk

Highlights

- S. aureus isolates from bulk tank milk and clinical mastitis had similar genetic background.

- Dairy cows can be carriers of subtypes that can cause clinical mastitis under right conditions.

- Three mobile genetic elements were primarily found among closely related ST151 isolates.

\begin{abstract}
Staphylococcus aureus is one of the most common pathogens that cause mastitis in dairy cows. Various subtypes, virulence genes and pathogenicity islands have been associated with isolates from bulk tank milk and clinical mastitis. So far, no Danish cattle associated S. aureus isolates have been wholegenome sequenced and further analyzed. Thus, the main objective was to investigate the population structure and genomic content of isolates from bulk tank milk and clinical mastitis, using whole-genome sequencing. This may reveal the origin of strains that cause clinical mastitis.

S. aureus isolates from bulk tank milk ( $\mathrm{n}=94)$ and clinical mastitis $(\mathrm{n}=63)$ were collected from 91 and 24 different farms, respectively and whole-genome sequenced. The genomic content was analyzed and a phylogenetic tree based on single nucleotide polymorphisms was constructed.

In general, the isolates from both bulk tank milk and clinical mastitis were of similar genetic background. This suggests that dairy cows are natural carriers of the $S$. aureus subtypes that cause clinical mastitis if the right conditions are present and that a broad range of subtypes cause mastitis. A phylogenetic cluster that mostly consisted of ST151 isolates carried three pathogenicity islands that were primarily found in this group. The prevalence of resistance genes was generally low. However, the first ST398 methicillin resistant S. aureus isolate from a Danish dairy cow with clinical mastitis was detected.
\end{abstract}


Keywords

Whole-genome sequencing, Staphylococcus aureus, bovine mastitis, bulk tank milk, population structure, virulence and resistance genes

\section{Introduction}

Staphylococcus aureus is an opportunistic pathogen that may cause severe infections in both humans and livestock and is a major cause of mastitis in dairy cows (Holmes and Zadoks, 2011)(Agersø et al., 2012)(Larsen et al., 2015). Bovine mastitis results in reduced animal welfare, milk quality and milk production which is the reason for remarkable economic losses worldwide (Halasa et al., 2007)(Haran et al., 2012)(Barkema et al., 2009). A variety of different sequence types (STs) (ST97, 126 133, 151, 479 and 771) (Holmes and Zadoks, 2011)(Zadoks et al., 2011) and spa-types (t518, t519, t524 t528, t529 and t543) have been associated with bovine mastitis and cattle worldwide (Hasman et al., 2010)(Ikawaty et al., 2009)(Sakwinska et al., 2011). Previous studies have shown that few types of strains belonging to specific genotypes are successful at causing persistent mastitis and strain RF122 (ST151) has been reported as one of the most common clone types involved in clinical mastitis (CM) (Kapur et al., 1995)(Reinoso et al., 2004)(Haveri et al., 2005)(Fritzgerald et al., 1997). This strain carries various mobile genetic elements (MGEs) that contain virulence genes and other types of genes related to host adaption. Most of these genes are found within specific types of MGEs known as S. aureus pathogenicity islands (SaPIs) (Herron-Olson et al., 2007). In general, various types of virulence genes have been detected in clinical and subclinical mastitis isolates and in bulk tank milk (BTM). These virulence factors are involved in: Host colonization (cap, clfA/B, cna, fib and sak), toxin production (tst, $s e a-j, h l a / b / g, l u k D / E / F S, e t A / B)$ and biofilm formation (icaD, fnbB) (Fueyo et al., 2005)(Bardiau et al., 2016)(Xu et al., 2015)(Fournier et al., 2008). Many of these virulence genes encode toxins that are also harmful to humans. For example, staphylococcal enterotoxins (encoded by se genes) cause food poisoning, the toxic shock syndrome toxin-1 (encoded by tst) causes toxic shock syndrome and leukocidins (encoded by $l u k D / E / F S$ ) are involved in various types of clinical infections (Asao et al., 2003)(Umeda et al., 2017)(Deurenberg et al., 2005) (Lina et al., 1999).

Methicillin resistant S. aureus (MRSA) belonging to ST398 has been observed among bovine mastitis isolates across the globe but has not disseminated among Danish herds of dairy cows (Holmes and Zadoks, 2011)(Zadoks et al., 2011). However, in Denmark this lineage has primarily been found in pigs and is now an increasing cause of human infections (Agers $\emptyset$ et al., 2012)(Larsen et al., 2015). Previously, various studies of Danish $S$. aureus isolates associated with bovine mastitis have been carried out (Katholm et al., 2012)(Aarestrup et al., 1995a)(H. D. Larsen et al., 2000)(Larsen et al., 2002)(Larsen et al., 2000) but no Danish isolates from BTM and CM have so far been whole-genome sequenced. Thus, the main objective of this study was to investigate the genomic content and population structure of Danish $S$. aureus isolates from BTM and CM, using whole-genome sequencing. A further objective was to investigate possible differences between the BTM and the CM isolates. 


\section{Materials and Methods}

\section{S. aureus isolates}

In 2016, CM isolates $(n=63)$ were all sampled from different cows on 24 different Danish farms. The aseptic foremilk samples were collected from dairy cows with CM according to the National Mastitis Council's guidelines. Samples of mastitis secrete or plates with growth were submitted to the Danish Veterinary Institute for S. aureus verification using Matrix-assisted laser desorption/ionization time of flight mass spectrometry (MALDI-TOF).

Isolates from BTM (n=94) were sampled from 91 different Danish dairy farms. The farms were selected based on the yearly BTM samples taken under a surveillance program for Streptococcus agalactiae as previously described (Katholm et al., 2012). Samples were analyzed with the Mastit4 real-time PCR test (DNA diagnostic A/S, Risskov, Denmark) at an analytic laboratory (Eurofins, Vejen, Denmark). Based on the PCR test result, 100 herds with the lowest Ct-value (ranging from 21-27) were selected and samples submitted to the Danish Veterinary Institute. The BTM samples were cultured by streaking 10 $\mu 1$ on blood agar (Columbia agar base (Oxoid, CM0331, Hampshire, UK) supplemented with 5\% calf blood) and on S. aureus selective ager (SA Select, bioMérieux, Marcy-l'Étoile, France). Colonies suspected for being $S$. aureus were further sub-cultured and verified as $S$. aureus using MALDI-TOF.

Both BTM and CM isolates were sampled from different farms distributed in all parts of the country.

DNA purification and whole-genome sequencing

S. aureus colonies were grown overnight on blood agar at $37^{\circ} \mathrm{C}$ and single colonies were cultured in 5 ml trypticase soy broth (Becton-Dickinson and Company, Franklin Lakes, USA) under the same conditions. DNA was purified using a Maxwell 16 LEV Blood DNA Kit (Promega, Madison, USA) according to manufacturer's instructions, with an additional lysis-phase including $200 \mu \mathrm{g} / \mathrm{ml}$ lysostaphin per sample (Sigma-Aldrich, St. Louis, USA). Subsequently, a Nextera XT kit (Illumina, San Diego, USA) was used for building DNA libraries according to manufacturer's instructions. The DNA libraries were paired-end sequenced applying Illumina's NextSeq platform with a read length on $2 \times 151 \mathrm{bp}$. The Illumina sequence reads have been deposited in NCBI's short read archive with the study accession no. SRP119902.

De novo assembly and subtyping

The quality of the Illumina raw reads was analyzed in FastQC 0.11 .5 and bases of low quality were trimmed in CLC bio’s Genomics Workbench (GW) v10.0 (CLCbio's, Aarhus, Denmark) using default settings. Subsequently, de novo assembly was performed in CLC bio's GW on default settings and a minimum contig size of 500 nt. MLST was performed at PubMLST (Jolley et al., 2004) and MLST v1.8 (Larsen et al., 2012) whereas spa-types were determined using spaTyper v1.0 (Bartels et al., 2014).

Identification of genomic content 
Resistance and virulence genes were identified in de novo assembled contigs using ResFinder v2.1 (Zankari et al., 2012) and VirulenceFinder v1.5 (Joensen et al., 2014), respectively. Few virulence genes ( fib, hla, icaD and $n u c$ ) were extracted from strain Sa52 (Ronco et al., 2017). Subsequently, the genes were identified in the assemblies using the BLASTN (Altschul et al., 1997) implementation in CLC bio's Main Workbench (MW) v7.7.3 and in general, if genes were located on > 1 contig CLC bio's MW was used to identify these. The presence of ORFs that belonged to eight different SaPIs (Table S1-S8) was investigated using CLC bio's GW.

\section{Statistics}

Statistical analyses were performed using GraphPad Prism v5.02 (GraphPad Software Inc., San Diego, USA). Differences in the presence of STs, spa-types and virulence/resistance genes between BTM and $\mathrm{CM}$ isolates were investigated applying a Chi-square test for independence. In cases of $\leq 5$ observations, a Fisher's exact test was used. The confidence interval was $95 \%$ and the difference considered significant when $P<0.05$.

Identification of single nucleotide polymorphisms

To investigate the relationship between the 157 isolates single nucleotide polymorphisms (SNPs) were identified using CSI Phylogeny v1.4 (Kaas et al., 2014) with S. aureus strain ED133 as reference chromosome (accession no. NC_017337). The SNPs were identified with a quality of $\geq 30$, a minimum depth of $\geq 10 \times$ and a distance between SNPs of $\geq 10$. Subsequently, a phylogenetic tree was visualized using iTOL v3.6.1 (Letunic and Bork, 2011).

\section{Results}

\section{MLST and spa-typing}

All isolates had an average sequencing depth of $>50$ fold except a single that had 47 fold. Statistical analyses showed no significant differences in distributions of STs or spa-types between BTM and CM isolates, except for ST1 and ST97 that were significantly associated with CM isolates (Table 1). Thirty different STs were found and 12 of these were new and subsequently registered at PubMLST (Jolley et al., 2004). Among BTM isolates 27 different STs were observed whereas 15 were found among the CM isolates. The most prevalent of the new STs were ST3891 and ST3897 found in 17\% (27/157) and 5\% $(8 / 157)$ of all isolates, respectively (Table 1). Of the remaining STs, the prevalence of the six most commonly found (ST50, 71, 97, 133, 151 and 479) ranged 5-19\% with ST151 as the most prevalent (Table 1). Among all isolates, 15 different spa-types were observed. However, 24 BTM and 15 CM isolates were identified as being of unknown spa-type. The prevalence of the six most often observed spa-types (t519, t524, t528, t529, t543 and t1403) ranged 5-27\%, with $\mathrm{t} 529$ as the most prevalent (Table 1)

Resistance and virulence genes 
In general, all genes were identified with thresholds of $90 \%$ nucleotide identity and $90 \%$ coverage of the query sequence length. Statistical analyses showed no significant differences in distributions of resistance genes between BTM and CM isolates. Ten different antibiotic resistance genes were observed. The norA gene was found in all isolates except a single one, whereas the second most prevalent resistance gene, blaZ was observed in 17\% (27/157) of the isolates. Only 9\% (14/157) of all isolates carried other types of resistance genes than blaZ and norA (Table 2). Altogether, 82\% (129/157) of all isolates carried no other resistance genes than norA (data not shown). Among 62 of the $63 \mathrm{CM}$ isolates only blaZ and norA were found whereas a single ST398 isolate carried a wide range of resistance genes (blaZ, ermB, lnuB, mecA, norA, tetK, and tetM) (data not shown)

Twenty-nine different virulence genes were identified and they could be divided into three groups according to prevalence among all 157 isolates. One group consisted of the 13 most prevalent genes (aur, hla, hlb $h \lg B, s p l A / B, \operatorname{lukD/E}, h \lg A / C, n u c, f i b$ and $i c a D$ ) found in $\geq 81 \%$ of all isolates. In the second group, the prevalence of six enterotoxin genes (seg, sei, sem, sen, seo and seu) ranged 45-69\%, whereas the prevalence of the ten remaining genes (sec, seh, sek, sel, seq, sea/sep, splE, tst, scn and sak) in the third group ranged 2-16\% (Table 2). According to statistical analyses five enterotoxin genes (sei, sem, sen, seo and seu) were significantly associated with BTM isolates whereas a serine protease gene (splE) and an enterotoxin gene (seh) were significantly associated with CM isolates (Table 2). When looking at the combination of virulence genes found among isolates within the eight most prevalent STs, no ST97 isolates carried any enterotoxin genes whereas $1 / 8$ of the ST71 and 1/14 of the ST133 isolates carried a single enterotoxin gene, sei (Table S9). The only types of enterotoxin genes that were found among the eight most prevalent STs were the six most prevalent types (seg, sei, sem, sen, seo and seu) (Table S9).

\section{Identification of SaPIs}

In seven different SaPIs, open reading frames (ORFs) were primarily identified with thresholds of > $80 \%$ nucleotide identity and $90 \%$ coverage of the query sequence length. In some cases the ORFs were identified with thresholds of $>70 \%$ nucleotide identity and a SaPI was only considered present if $\geq 80 \%$ of its ORFs were present. Our analyses showed that some types of SaPIs were primarily present among isolates with closely related genetic background (Table 3 ). The three SaPIs; $\varphi 12$ bov, $v$ SaBov and $\varphi \mathrm{SaBov}-v-\mathrm{Sa} \beta \varphi$ were only identified among a group of closely related isolates that primarily belonged to $\mathrm{ST} 151$, except for $v \mathrm{SaBov}$ that was also found in a single ST7 isolate. Isolates in this group that did not belong to ST151 were either single locus variants (SLVs) (ST3899, 3900 and 705) or double locus variants (DLVs) (ST504) of ST151. Additionally, SaPIbov1 and SaPIbov-vSa $\alpha$ were primarily found in six ST504 isolates but also in a single ST705 and a single ST71 isolate. In contrast, SaPIbov4 and SaPIbov5 were found among isolates from many different STs that were not closely related and none of the 157 isolates carried SaPIbov2 (Table 3). A visual overview of the distribution of SaPIs among all isolates can be found in Fig 1.

Phylogenetic analysis 
The SNP analysis included 38782 variant positions and $67.8 \%$ of the reference chromosome was covered by all isolates. In general, the phylogenetic tree showed that both CM and BTM isolates clustered together into groups of identical or closely related STs (Fig 1). The largest cluster in the tree consisted primarily of 30 ST151 isolates whereas the remaining isolates were either SLVs (ST3899, 3900 and 705) or DLVs (ST504) of ST151. The second largest cluster consisted of 41 isolates whereof eight belonged to ST50 and the post prevalent of the remaining new STs were 26 ST3891 isolates (SLVs of ST50). The third largest cluster consisted of nine ST479 isolates, eight ST3897 isolates and five ST1380 isolates. Furthermore, smaller clusters primarily including isolates that belonged to ST133, ST97 and ST71 were present (Fig 1).

\section{Discussion}

Here, we carried out whole-genome sequencing to investigate the population structure and genomic content of 157 Danish S. aureus isolates from BTM and dairy cows with CM. To our knowledge it is the first time that such types of Danish isolates have been whole-genome sequenced and made publicly available. Statistical analyses showed no significant differences in the distribution of spa-types or STs between the two isolate groups except for ST97 and ST1 that were significantly more associated with CM isolates compared to BTM isolates (Table 1). Only four of all isolates belonged to ST1 and therefore it is difficult to conclude further on this finding. Isolates from BTM samples may originate from subclinical infected quarters, but also from extra-mammary sites such as teat skin, teat canal and the cow environment or from milking staff (Haveri et al., 2008). This could be the reason for finding a more diverse composition of STs in BTM samples (27 different STs) compared to CM samples (15 different STs). Furthermore, the BTM isolates were collected from farms that had shown an increased concentration of $S$. aureus in BTM (PCR Ct-values: 21-27). A study suggests that Ct-values < 32 very likely can be interpreted as reflecting S. aureus intra-mammary infections (Mahmmod et al., 2017). Therefore, it is likely that the isolates from BTM were partly from cows with subclinical mastitis which is a mild form of mastitis that requires further testing to be recognized by the farmer. It has been described that a high strain heterogeneity can be interpreted as evidence of environmental mastitis (Klaas and Zadoks, 2017). Thus, the BTM isolates that showed increased strain heterogeneity compared to the $\mathrm{CM}$ isolates, could be associated with environmental mastitis

The phylogenetic analysis showed that the BTM isolates clustered together with CM isolates of identical or closely related STs. A large cluster of isolates that primarily belonged to ST151 was observed and the majority of the isolates in this cluster carried three SaPIs $(\varphi 12$ bov, $v \operatorname{SaBov}$ and $\varphi \operatorname{SaBov}-v-\mathrm{Sa} \beta \varphi)$ found in strain RF122 (Herron-Olson et al., 2007). These three SaPIs were exclusively found in this cluster except for a single ST7 isolate that also carried one of these SaPIs (Fig 1). Strain RF122 belonged to ST151 and has been reported to be a commonly observed mastitis causing clone type (Fritzgerald et al., 1997). The SaPIs originating from RF122 contained various virulence genes and therefore it has been suggested the these SaPIs play an important role regarding the CM pathogenesis and successful adaption to dairy cows (Herron-Olson et al., 2007). SaPI $\varphi \mathrm{SaBov-vSa} \beta \varphi$ carries leucocidin genes (lukE/D), serine proteases $(\operatorname{splC} / E / F)$ and enterotoxin genes $(\mathrm{sec} / \mathrm{g} / \mathrm{i} / \mathrm{m} / \mathrm{n} / \mathrm{o})$ whereas $v \mathrm{SaBov}$ carries streptolycin genes 
(Table S6 and S7). The streptolycin genes encode leucocidin homologs that originates from Streptococcus pyogenes (Herron-Olson et al., 2007) and many of the virulence genes carried by $\varphi \mathrm{SaBov}-\imath \mathrm{Sa} \beta \varphi$ have previously been found among mastitis isolates (Fueyo et al., 2005)(Bardiau et al., 2016)(Fournier et al., 2008)(Kot et al., 2016). Additionally, these three SaPIs ( $\varphi$ 12bov, $v$ SaBov and $\varphi \mathrm{SaBov}-v-\mathrm{Sa} \beta \varphi$ ) contain many hypothetical and phage related genes (Table S6-S8) that encode proteins of unknown functions and further studies could reveal which potential role they play. Both statistical and phylogenetic analyses showed that the BTM and CM isolates in general were of identical genetic background. This correspond to other studies (Boss et al., 2016)(Conceicão, 2017)(Jørgensen et al., 2005) which found that STs and spa-types that were often associated with bovine mastitis are also present in healthy cows and BTM. These findings indicate that dairy cows are natural carriers of $S$. aureus subtypes that can cause CM, for example if the cows appear immunocompromised combined with poor milking practices and hygiene etc.

Some of the most prevalent STs (ST97, 133, 151 and 479) found in this study have previously been associated with bovine mastitis (Holmes and Zadoks, 2011)(Zadoks et al., 2011)(Boss et al., 2016) whereas others (ST50 and 71) have been related to healthy cows and BTM (Smith et al., 2005)(Hata et al., 2010). In addition, the two most prevalent of the 12 new STs ST3891 and ST3897 were SLVs of ST50 and ST479, respectively. The most prevalent spa-type was t529 and observed in 27\% (43/157) of the isolates, followed by t1403 and t519 that were both found in 10\% (16/157) of the isolates. These three spa-types have all been associated with bovine mastitis (Ikawaty et al., 2009)(Sakwinska et al., 2011)(Boss et al., 2016)(Johler et al., 2011) but also healthy cows (Hasman et al., 2010). All 30 ST151 isolates belonged to spa-type t529 which correspond to a previous study (Sakwinska et al., 2011). Many isolates (39/157) were identified as being of unknown spa-type using spaTyper (Bartels et al., 2014). The main reason for this was that the spa genes were located on $>1$ contig and therefore not all repeats were identified by spaTyper (Bartels et al., 2014). Assembly and sequencing error could also explain why the order of the spa repeats was not determined correctly. However, it was beyond the scope of this study to register new spa-types according to guidelines found at the Ridom SapServer (http://www.spaserver.ridom.de/).

In general, the prevalence of resistance genes was low which correspond to a previous study of Danish mastitis isolates where $81 \%$ of 105 isolates were susceptible to 11 antibiotics (Aarestrup et al., 1995a). However, the first ST398 livestock-associated (LA) MRSA isolate (Sa52) from a Danish dairy cow with CM was discovered. The fact that strain Sa52 carried many other resistance genes than the rest of the CM isolates that only carried blaZ or norA, indicates that it has been transmitted to the dairy cow from an environment with a different selective pressure in regard to antibiotics. Interestingly, previous studies suggest the ST398 lineage has the ability to efficiently jump between humans and livestock and cause severe humans infections (Larsen et al., 2015)(Price et al., 2012). Additionally, it has been suggested that human epidemic MRSA clones originate from isolates within CC97 that have jumped from cows to humans (Spoor et al., 2013). This makes it important to further monitor cattle herds to avoid potential problems regarding LA-MRSA with zoonotic potential, even though the transmission of for example 
ST398 strains between humans and dairy cows seems to occur less frequently (Sakwinska et al., 2011)(Boss et al., 2016). LA-MRSA isolates belonging to ST398 have primarily been associated with pigs but strain Sa52 was sampled on a farm where no pig farming had taken place (Larsen et al., 2015)(Price et al., 2012). Thus, it is unlikely that this strain was directly related to pig farming but it could have been transmitted by a visitor or farm worker carrying the clone. Seven-teen $\%$ of all isolates carried blaZ which correspond to a previous Danish study where $17 \%$ of 105 isolates produced betalactamases (Aarestrup et al., 1995a). Remarkably, norA was found in all isolates except a single one. This gene encodes a multidrug drug resistance efflux pump that mediates resistance to quinolones and a variety of other antiseptic compounds (Santos Costa et al., 2015)(Kaatz and Seo, 1995). Since fluorquinolones are not used to treat Danish dairy cows for CM (https://www.foedevarestyrelsen.dk/Leksikon/Sider/VetStat.aspx) the presence of this gene must be driven by other factors. It may be suggested that norA caused resistance to antiseptic compounds used to increase the hygiene in the Danish dairy industry.

Six enterotoxin genes ( $\mathrm{seg}, \mathrm{sei}, \mathrm{sem}, \mathrm{sen}, \mathrm{seo}$ and $\mathrm{seu}$ ) were found more frequently than the rest of the enterotoxin genes and were additionally the only types found among isolates from the eight most prevalent STs (Table S9). Five of these genes were significantly more associated with BTM isolates compared to CM isolates. Previously, many of these genes have been found in S. aureus isolates from bovine mastitis (Fueyo et al., 2005)(Xu et al., 2015)(Fournier et al., 2008)(Kot et al., 2016). However, the role of enterotoxins in the mastitis pathogenesis is not clear and studies indicate that they are not essential (Larsen et al., 2002)(Larsen et al., 2000). Enterotoxins are heat-stabile and may therefore be found in various dairy products such as milk after heat treatment (Jørgensen et al., 2005)(Hennekinne et al., 2012). Previously, enterotoxins have been reported to be associated with staphylococcal food poisoning caused by cow milk or other dairy products and even with mastitis in humans (Asao et al., 2003)(Jørgensen et al., 2005)(Hennekinne et al., 2012)(Franck et al., 2017). Interestingly, isolates from the highly prevalent ST97 and ST133 that have been found to be strongly associated with CM (Holmes and Zadoks, 2011)(Zadoks et al., 2011), carried almost no enterotoxins genes (Table S9). Concordantly, a previous PCR investigation of 106 Danish S. aureus isolates from subclinical and CM showed that none of the isolates carried any enterotoxin genes (Aarestrup et al., 1995b). Currently, more than 20 types of enterotoxin genes have been identified (Hennekinne et al., 2012) but in this study only 12 types were investigated.Thus, it is possible that the isolates carried other enterotoxin genes than those investigated. Furthermore, a high proportion $(\geq 81 \%)$ of all isolates carried the leukotoxin encoding genes $l u k D$ and $l u k E$. These genes are often found in isolates associated with bovine mastitis (Fueyo et al., 2005)(Bardiau et al., 2016) but have also been detected in clinical isolates from humans (Yoong and Torres, 2014).

\section{Conclusion}

In summary, both statistical and phylogenetic analyses showed that isolates from BTM and CM generally were of similar genetic background. This suggests that dairy cows can be natural carriers of, or subclinicallly infected with, $S$. aureus subtypes that can cause CM if the right conditions are present. A 
large cluster primarily consisting of ST151 isolates carried three SaPIs that were almost only found in this group and probably are involved in host adaption and the mastitis pathogenesis. A high proportion of all isolates carried leukotoxin genes and other toxin genes whereas five enterotoxin genes were significantly more associated with BTM isolates compared to CM isolates. Thus, both BTM and CM isolates carried genes that encode toxins that are harmful to humans. The prevalence of resistance genes was in general low but the first ST398 LA-MRSA isolate from a Danish dairy cow with CM was detected. Further surveillance of the Danish dairy cows is therefore important in order to avoid dissemination of zoonotic pathogens.

\section{Conflicts of interest statement}

All authors declare no conflicts of interest

\section{Acknowledgement}

This project was supported by grants from Promilleafgiftsfonden and the Danish Milk Levy Fund (Mælkeafgiftsfonden). This project was also a part of the "STOPMAST" project. Furthermore, we would like to thank Mette T. Christiansen from Statens Serum Institut and Kári K. Mouritsen from the Technical University of Denmark for technical assistance.

\section{References}

Agersø, Y., Hasman, H., Cavaco, L.M., Pedersen, K., Aarestrup, F.M., 2012. Study of methicillin resistant Staphylococcus aureus (MRSA) in Danish pigs at slaughter and in imported retail meat reveals a novel MRSA type in slaughter pigs. Vet. Microbiol. 157, 246-250. doi:10.1016/j.vetmic.2011.12.023

Altschul, S.F., Madden, T.L., Schäffer, A.A., Zhang, J., Zhang, Z., Miller, W., Lipman, D.J., 1997. Gapped BLAST and PSI-BLAST:a new generation of protein database search programs. Nucleic Acids Res 25, 3389-3402. doi:10.1093/nar/25.17.3389

Asao, T., Kumeda, Y., Kawai, T., Shibata, T., Oda, H., Haruki, K., Nakazawa, H., Kozaki, S., 2003. An extensive outbreak of staphylococcal food poisoning due to low-fat milk in Japan: estimation of enterotoxin $\mathrm{A}$ in the incriminated milk and powdered skim milk. Epidemiol. Infect. 130, 33-40. doi:10.1017/S0950268802007951

Bardiau, M., Caplin, J., Detilleux, J., Graber, H., Moroni, P., Taminiau, B., Mainil, J.G., 2016. Existence of two groups of Staphylococcus aureus strains isolated from bovine mastitis based on biofilm formation, intracellular survival, capsular profile and agr-typing. Vet. Microbiol. 185, 1-6. doi:10.1016/j.vetmic.2016.01.003

Barkema, H.W., Green, M.J., Bradley, A.J., Zadoks, R.N., 2009. The role of contagious disease in udder health. J. Dairy Sci. 92, 4717-4729. doi:10.3168/jds.2009-2347.The

Bartels, M.D., Petersen, A., Worning, P., Nielsen, J.B., Larner-Svensson, H., Johansen, H.K., Andersen, L.P., Jarløv, J.O., Boye, K., Larsen, A.R., Westh, H., 2014. Comparing whole-genome sequencing 
with sanger sequencing for spa typing of methicillin-resistant Staphylococcus aureus. J. Clin. Microbiol. 52, 4305-4308. doi:10.1128/JCM.01979-14

Boss, R., Cosandey, A., Luini, M., Artursson, K., Bardiau, M., Al, E., 2016. Bovine Staphylococcus aureus : Subtyping, evolution, and zoonotic transfer. J. Dairy Sci. 515-528.

Conceicão, T., 2017. Healthy Bovines as Reservoirs of Major Pathogenic Lineages of Staphylococcus aureus in Portugal. Microb. drug Resist. 0, 1-7. doi:10.1089/mdr.2017.0074

Cucarella, C., Solano, C., Valle, J., Amorena, B., Lasa, I.G.O., Penade, R., 2001. Bap , a Staphylococcus aureus surface protein involved in biofilm formation. J. Bacteriol. 183, 2888-2896. doi:10.1128/JB.183.9.2888

Cucarella, C., Tormo, M.Á., Úbeda, C., Trotonda, M.P., Monzón, M., Peris, C., Amorena, B., Lasa, Í., Penadés, J.R., 2004. Role of Biofilm-Associated Protein Bap in the Pathogenesis of Bovine Staphylococcus aureus. Infect. Immun. 72, 2177-2185. doi:10.1128/IAI.72.4.2177-2185.2004

Deurenberg, R.H., Nieuwenhuis, R.F., Driessen, C., London, N., Stassen, F.R., Tiel, F.H. Van, Stobberingh, E.E., Vink, C., 2005. The prevalence of the Staphylococcus aureus tst gene among community- and hospital-acquired strains and isolates from Wegener's Granulomatosis patients. FEMS Microbiol. Lett. 245, 185-189. doi:10.1016/j.femsle.2005.03.002

Fitzgerald, J.R., Monday, S.R., Foster, T.J., Bohach, G.A., Hartigan, P.J., Meaney, W.J., Smyth, C.J., 2001. Characterization of a putative pathogenicity island from bovine Staphylococcus aureus encoding multiple superantigens. J. Bacteriol. 183, 63-70. doi:10.1128/JB.183.1.63

Fournier, C., Kuhnert, P., Frey, J., Miserez, R., Kirchhofer, M., Kaufmann, T., Steiner, A., Graber, H.U., 2008. Bovine Staphylococcus aureus: Association of virulence genes, genotypes and clinical outcome. Res. Vet. Sci. 85, 439-448. doi:10.1016/j.rvsc.2008.01.010

Franck, K.T., Gumpert, H., Olesen, B., Larsen, A.R., Petersen, A., Bangsborg, J., Albertsen, P., Westh, H., 2017. Staphylococcal aureus Enterotoxin C and Enterotoxin-Like L Associated with Postpartum Mastitis. Front. Microbiol. 8, 1-5. doi:10.3389/fmicb.2017.00173

Fritzgerald, J.R., Meaney, W.J., Hartigan, P.J., Smyth, C.J., Kapur, V., 1997. Fine-structure molecular epidemiological analysis of Staphylococcus aureus recovered from cows. Epidemiol. Infect. 261269.

Fueyo, J.M., Mendoza, M.C., Rodicio, M.R., Muñiz, J., Alvarez, M.A., Martín, M.C., 2005. Cytotoxin and pyrogenic toxin superantigen gene profiles of Staphylococcus aureus associated with subclinical mastitis in dairy cows and relationships with macrorestriction genomic profiles. J. Clin. Microbiol. 43, 1278-1284. doi:10.1128/JCM.43.3.1278-1284.2005

Halasa, T., Huijps, K., Østerås, O., Hogeveen, H., 2007. Economic effects of bovine mastitis and mastitis management: A review. Vet. Q. 29, 18-31. doi:10.1080/01652176.2007.9695224

Haran, K.P., Godden, S.M., Boxrud, D., Jawahir, S., Bender, J.B., Sreevatsan, S., 2012. Prevalence and 
characterization of Staphylococcus aureus, including methicillin-resistant Staphylococcus aureus, isolated from bulk tank milk from Minnesota dairy farms. J. Clin. Microbiol. 50, 688-695. doi:10.1128/JCM.05214-11

Hasman, H., Moodley, A., Guardabassi, L., Stegger, M., Skov, R.L., Aarestrup, F.M., 2010. spa type distribution in Staphylococcus aureus originating from pigs, cattle and poultry. Vet. Microbiol. 141, 326-331. doi:10.1016/j.vetmic.2009.09.025

Hata, E., Katsuda, K., Kobayashi, H., Uchida, I., Tanaka, K., Eguchi, M., 2010. Genetic variation among Staphylococcus aureus strains from bovine milk and their relevance to methicillin-resistant isolates from humans. J. Clin. Microbiol. 48, 2130-2139. doi:10.1128/JCM.01940-09

Haveri, M., Hovinen, M., Roslo, A., Pyo, S., 2008. Molecular types and genetic profiles of Staphylococcus aureus strains isolated from bovine intramammary infections and extramammary sites. J. Clin. Microbiol. 46, 3728-3735. doi:10.1128/JCM.00769-08

Haveri, M., Taponen, S., Pyo, S., 2005. Bacterial genotype affects the manifestation and persistence of bovine Staphylococcus aureus intramammary infection. J. Clin. Microbiol. 43, 959-961. doi:10.1128/JCM.43.2.959

Hennekinne, J., Buyser, M. De, Dragacci, S., 2012. Staphylococcus aureus and its food poisoning toxins: characterization and outbreak investigation. Fems Microb. Rev 36, 815-836. doi:10.1111/j.1574-6976.2011.00311.x

Herron-Olson, L., Fitzgerald, J.R., Musser, J.M., Kapur, V., 2007. Molecular correlates of host specialization in Staphylococcus aureus. PLoS One 2(10), e1120.

Holmes, M.A., Zadoks, R.N., 2011. Methicillin resistant S. aureus in human and bovine mastitis. J. Mammary Gland Biol. Neoplasia 16, 373-382. doi:10.1007/s10911-011-9237-x

Ikawaty, R., Brouwer, E.C., Jansen, M.D., Duijkeren, E. Van, Mevius, D., Verhoef, J., Fluit, A.C., 2009. Characterization of Dutch Staphylococcus aureus from bovine mastitis using a Multiple Locus Variable Number Tandem Repeat Analysis. Vet. Microbiol. 136, 277-284. doi:10.1016/j.vetmic.2008.10.034

Joensen, K.G., Scheutz, F., Lund, O., Hasman, H., Kaas, R.S., Nielsen, E.M., Aarestrup, F.M., 2014. Real-time whole-genome sequencing for routine typing, surveillance, and outbreak detection of verotoxigenic Escherichia coli. J. Clin. Microbiol. 52, 1501-1510. doi:10.1128/JCM.03617-13

Johler, S., Layer, F., Stephan, R., 2011. Comparison of Virulence and Antibiotic Resistance Genes of Food Poisoning Outbreak Isolates of Staphylococcus aureus with Isolates Obtained from Bovine Mastitis Milk and Pig Carcasses. J. Food Prot. 74, 1852-1859. doi:10.4315/0362-028X.JFP-11-192

Jolley, K.A., Chan, M.-S., Maiden, M.C.J., 2004. mlstdbNet - distributed multi-locus sequence typing (MLST) databases. BMC Bioinformatics 5, 86. doi:10.1186/1471-2105-5-86

Jørgensen, H.J., Mørk, T., Rørvik, L.M., 2005. The Occurrence of Staphylococcus aureus on a Farm 
with Small-Scale Production of Raw Milk Cheese. J. Dairy Sci. 88, 3810-3817. doi:10.3168/jds.S0022-0302(05)73066-6

Kapur, V., Sischo, W.M., Greer, R.S., Whittam, T.S., Musser, J.M., 1995. Molecular population genetic analysis of staphylococcus aureus recovered from cows. J. Clin. Microbiol. 33, 376-380.

Katholm, J., Bennedsgaard, T.W., Koskinen, M.T., Rattenborg, E., 2012. Quality of bulk tank milk samples from Danish dairy herds based on real-time polymerase chain reaction identification of mastitis pathogens. J. Dairy Sci. 95, 5702-8. doi:10.3168/jds.2011-5307

Klaas, I.C., Zadoks, R.N., 2017. An update on environmental mastitis: Challenging perceptions. Transbound Emerg Dis. 1-20. doi:10.1111/tbed.12704

Kot, B., Szweda, P., Frankowska-Maciejewska, A., Piechota, M., Wolska, K., 2016. Virulence gene profiles in Staphylococcus aureus isolated from cows with subclinical mastitis in eastern Poland. J. Dairy Res. 83, 228-235. doi:10.1017/S002202991600008X

Kaas, R.S., Leekitcharoenphon, P., Aarestrup, F.M., Lund, O., 2014. Solving the problem of comparing whole bacterial genomes across different sequencing platforms. PLoS One 9, 1-8. doi:10.1371/journal.pone.0104984

Kaatz, G.W., Seo, S.M., 1995. Inducible NorA-Mediated Multidrug Resistance in Staphylococcus aureus. Antimicrob. Agents Chemother. 39, 2650-2655.

Larsen, H.D., Sloth, K.H., Elsberg, C., Enevoldsen, C., 2000. The dynamics of Staphylococcus aureus intramammary infection in nine Danish dairy herds. Vet. Microbiol. 71, 89-101.

Larsen, H.D., Aarestrup, F.M., Jensen, N.E., 2002. Geographical variation in the presence of genes encoding superantigenic exotoxins and beta-hemolysin among Staphylococcus aureus isolated from bovine mastitis in Europe and USA. Vet. Microbiol. 85, 61-67.

Larsen, J., Petersen, A., Sørum, M., Stegger, M., Van Alphen, L., Valentiner-Branth, P., Knudsen, L.K., Larsen, L.S., Feingold, B., Price, L.B., Andersen, P.S., Larsen, A.R., Skov, R.L., 2015. Meticillinresistant staphylococcus aureus CC398 is an increasing cause of disease in people with no livestock contact in Denmark, 1999 to 2011. Eurosurveillance 20. doi:10.2807/15607917.ES.2015.20.37.30021

Larsen, Huda, A., Eriksen, N.H.R., Jensen, N.E., 2000. Differences between Danish bovine and human Staphylococcus aureus isolates in possession of superantigens. Vet. Microbiol. 76, 153-162.

Larsen, M. V., Cosentino, S., Rasmussen, S., Friis, C., Hasman, H., Marvig, R.L., Jelsbak, L., SicheritzPonten, T., Ussery, D.W., Aarestrup, F.M., Lund, O., 2012. Multilocus Sequence Typing of TotalGenome-Sequenced Bacteria. J. Clin. Microbiol. 50, 1355-1361. doi:10.1128/JCM.06094-11

Letunic, I., Bork, P., 2011. Interactive Tree Of Life v2: online annotation and display of phylogenetic trees made easy. Nucleic Acids Res. 39, W475-W478. doi:10.1093/nar/gkr201 
Lina, G., Piémont, Y., Godail-gamot, F., Peter, M., 1999. Involvement of Panton-valentine leukocidin producing Staphylococcus aureus in primary skin infections and pneumonia. Clin. inectious Dis. $1128-1132$.

Mahmmod, Y.S., Klaas, I.C., Enevoldsen, C., 2017. DNA carryover in milk samples from routine milk recording used for PCR-based diagnosis of bovine Staphylococcus aureus mastitis. J. Dairy Sci. 100, 5709-5716. doi:10.3168/jds.2016-12330

Price, L.B., Stegger, M., Hasman, H., Aziz, M., Larsen, J., Andersen, S., Pearson, T., Laurent, F., Keim, P., Skov, R., Aarestrup, F.M., 2012. Staphylococcus aureus CC398: Host Adaptation and Emergence of Methicillin Resistance in Livestock. MBio 3(1), e00305-11.

Reinoso, E., Bettera, S., Frigerio, C., Direnzo, M., Calzolari, A., Bogni, C., 2004. RAPD-PCR analysis of Staphylococcus aureus strains isolated from bovine and human hosts. Microbiol. Res. 159, 245255. doi:10.1016/j.micres.2004.04.002

Ronco, T., Stegger, M., Pedersen, K., 2017. Draft Genome Sequence of a Sequence Type 398 Methicillin-Resistant Staphylococcus aureus Isolate from a Danish Dairy Cow with Mastitis. Genome Announc. 5, e00492-17.

Sakwinska, O., Giddey, M., Moreillon, M., Morisset, D., Waldvogel, A., Moreillon, P., 2011. Staphylococcus aureus host range and human-bovine host shift. Appl. Environ. Microbiol. 77, 5908-5915. doi:10.1128/AEM.00238-11

Santos Costa, S., Viveiros, M., Rosato, A.E., Melo-Cristino, J., Couto, I., 2015. Impact of efflux in the development of multidrug resistance phenotypes in Staphylococcus aureus. BMC Microbiol. 15, 232-247. doi:10.1186/s12866-015-0572-8

Smith, E.M., Green, L.E., Medley, G.F., Bird, H.E., Fox, L.K., Schukken, Y.H., Kruze, J. V, Bradley, a J., Zadoks, R.N., Dowson, C.G., 2005. Multilocus sequence typing of intercontinental bovine Staphylococcus aureus isolates. J. Clin. Microbiol. 43, 4737-4743. doi:10.1128/JCM.43.9.4737

Spoor, L.E., Mcadam, P.R., Weinert, L.A., Rambaut, A., Hasman, H., Aarestrup, F.M., Kearns, A.M., Larsen, A.R., Skov, R.L., Ross, J., 2013. Livestock Origin for a Human Pandemic Clone of Community- Associated Methicillin-Resistant Staphylococcus aureus. MBio 4, e00356-13. doi:10.1128/mBio.00356-13.Editor

Umeda, K., Nakamura, H., Yamamoto, K., Nishina, N., Yasufuku, K., Hirai, Y., Hirayama, T., Goto, K., Hase, A., Ogasawara, J., 2017. Molecular and epidemiological characterization of staphylococcal foodborne outbreak of Staphylococcus aureus harboring seg, sei, sem, sen, seo, and selu genes without production of classical enterotoxi. Int. J. Food Microbiol. 256, 30-35. doi:10.1016/j.ijfoodmicro.2017.05.023

Viana, D., Blanco, J., Tormo-más, M.Á., Selva, L., Guinane, C.M., Baselga, R., Corpa, J.M., Lasa, Í., Novick, R.P., Fitzgerald, J.R., Penadés, J.R., 2010. Adaptation of Staphylococcus aureus to ruminant and equine hosts involves SaPI-carried variants of von Willebrand factor-binding protein. 
Mol. Biol. Evol. 77, 1583-1594. doi:10.1111/j.1365-2958.2010.07312.x

Xu, J., Tan, X., Zhang, X., Xia, X., Sun, H., 2015. The diversities of staphylococcal species, virulence and antibiotic resistance genes in the subclinical mastitis milk from a single Chinese cow herd. Microb. Pathog. 88, 29-38. doi:10.1016/j.micpath.2015.08.004

Yoong, P., Torres, V.J., 2014. The effects of Staphylococcus aureus leukotoxins on the host: cell lysis and beyond. Curr Opin Microbiol 16, 63-69. doi:10.1016/j.mib.2013.01.012.The

Zadoks, R.N., Middleton, J.R., McDougall, S., Katholm, J., Schukken, Y.H., 2011. Molecular epidemiology of mastitis pathogens of dairy cattle and comparative relevance to humans. J. Mammary Gland Biol. Neoplasia 16, 357-372. doi:10.1007/s10911-011-9236-y

Zankari, E., Hasman, H., Cosentino, S., Vestergaard, M., Rasmussen, S., Lund, O., Aarestrup, F.M., Larsen, M.V., 2012. Identification of acquired antimicrobial resistance genes. J. Antimicrob. Chemother. 67, 2640-2644. doi:10.1093/jac/dks261

Aarestrup, Wegener, H.C., Rosdahl, V.T., 1995a. Evaluation of phenotypic and genotypic methods for epidemiological typing of Staphylococcus aureus isolates from bovine mastitis in Denmark. Vet. Microbiol. 45, 139-150. doi:10.1016/0378-1135(95)00043-A

Aarestrup, Andersen, J.K., Jensen, N.E., 1995b. Lack of Staphylococcal enterotoxin production among strains of Staphylococcus aureus from bovine mastitis in Denmark. Acta Vet Scand 36, 273-275. 
Fig1 Phylogenetic tree including the distribution of SaPIs

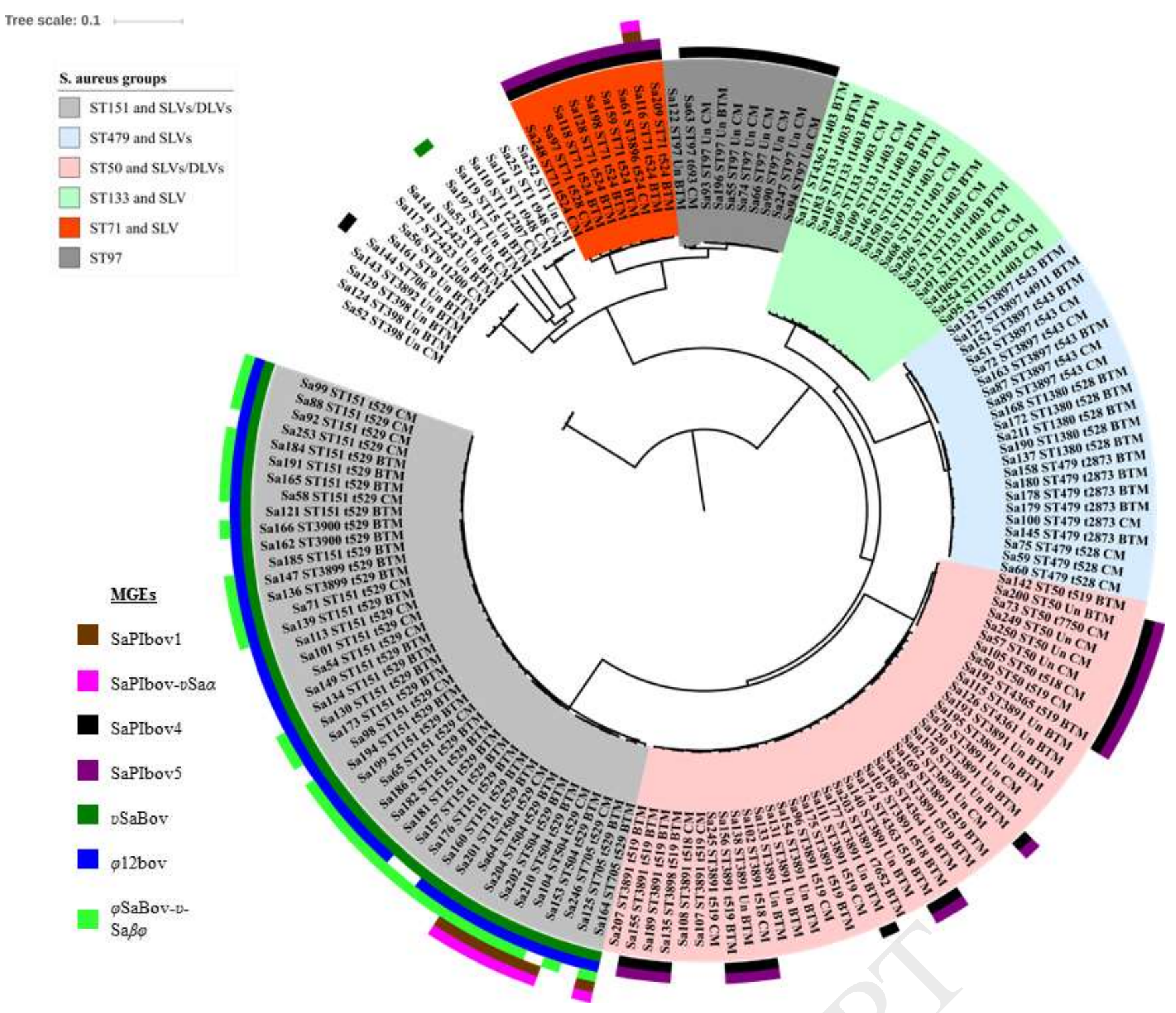

The phylogenetic tree is based on 38782 SNPs and S. aureus strain ED133 was used as reference chromosome. It shows that the 157 S. aureus isolates from bulk tank milk (BTM) and clinical mastitis (CM) cluster together into groups of closely related STs. Additionally, the distribution of seven different S. aureus pathogenicity Islands (SaPIs) is shown as colored strips. Three different SaPIs (vSaBov and $\varphi \mathrm{SaBov}-v-\mathrm{Sa} \beta \varphi$ ) were only found in a cluster that primarily consisted of ST151 isolates (marked in light gray). This cluster included single locus variants (SLVs) (ST3899, 3900 and 705) and double locus variants (DLVs) (ST504) of ST151. Only a single ST7 isolate outside this cluster carried $v$ SaBov. In contrast, SaPIbov4 and SaPIbov5 were found among various STs that were not all closely related. 
Table 1 Prevalence of STs and spa-types among S. aureus isolates

\begin{tabular}{|c|c|c|c|c|c|c|c|}
\hline $\begin{array}{l}\text { ST } \\
(\%)\end{array}$ & $\begin{array}{l}\mathrm{CM} \\
(\mathrm{n}=63)\end{array}$ & $\begin{array}{l}\text { BTM } \\
(\mathrm{n}=94)\end{array}$ & $P$-value & $\begin{array}{l}\text { spa-type } \\
(\%)\end{array}$ & $\begin{array}{l}\mathrm{CM} \\
(\mathrm{n}=63)\end{array}$ & $\begin{array}{l}\text { BTM } \\
(\mathrm{n}=94)\end{array}$ & $P$-value \\
\hline 151 (19) & 11 & 19 & 0.6672 & $\mathbf{t 5 2 9}(27)$ & 14 & 29 & 0.2347 \\
\hline 3891* (17) & 8 & 19 & 0.2213 & t519 (10) & 6 & 10 & 0.8210 \\
\hline 133 (9) & 9 & 5 & 0.0838 & $\mathbf{t} 1403$ & 9 & 7 & 0.1650 \\
\hline $97(6)$ & 8 & 2 & 0.0151 & $\mathbf{t 5 2 8}(6)$ & 4 & 5 & 1.0000 \\
\hline $479(6)$ & 4 & 5 & 1.0000 & t524 (5) & 2 & 6 & 0.4768 \\
\hline $50(5)$ & 6 & 2 & 0.0608 & t543 (5) & 4 & 3 & 0.4395 \\
\hline $71(5)$ & 2 & 6 & 0.4768 & $\mathrm{t} 2873$ & 1 & 5 & 0.4027 \\
\hline $3897 *(5)$ & 4 & 4 & 0.7146 & $\mathrm{t} 518$ & 2 & 3 & 1.0000 \\
\hline $504(4)$ & 2 & 4 & 1.0000 & t693 (1) & 1 & 0 & 0.4013 \\
\hline $1(3)$ & 4 & 0 & 0.0245 & t948 (1) & 2 & 0 & 0.1595 \\
\hline $1380(3)$ & 0 & 5 & 0.0831 & $\mathrm{t} 1200$ & 1 & 0 & 0.4013 \\
\hline $398(2)$ & 1 & 2 & 1.0000 & $\mathrm{t} 2207$ & 1 & 0 & 0.4013 \\
\hline $705(2)$ & 1 & 2 & 1.0000 & $\mathrm{t} 4911$ (1) & 0 & 1 & 1.0000 \\
\hline $7(1)$ & 0 & 1 & 1.0000 & $\mathrm{t} 7652$ & 0 & 1 & 1.0000 \\
\hline $8(1)$ & 1 & 0 & 0.4013 & $\mathrm{t} 7750$ & 1 & 0 & 0.4013 \\
\hline $9(1)$ & 1 & 1 & 1.0000 & Unk (25) & 15 & 24 & 0.8066 \\
\hline $15(1)$ & 0 & 1 & 1.0000 & & & & \\
\hline $132(1)$ & 0 & 1 & 1.0000 & & & & \\
\hline $706(1)$ & 0 & 1 & 1.0000 & & & & \\
\hline $2423(1)$ & 0 & 2 & 0.5164 & & & & \\
\hline $3892 *(1)$ & 0 & 1 & 1.0000 & & & & \\
\hline $3896^{*}(1)$ & 1 & 0 & 0.4013 & & & & \\
\hline $3898 *(1)$ & 0 & 2 & 0.5164 & & & & \\
\hline $3899 *(1)$ & 0 & 2 & 0.5164 & & & & \\
\hline $3900 *(1)$ & 0 & 2 & 0.5164 & & & & \\
\hline $4361 *(1)$ & 0 & 1 & 1.0000 & & & & \\
\hline $4362 *(1)$ & 0 & 1 & 1.0000 & & & & \\
\hline $4363 *(1)$ & 0 & 1 & 1.0000 & & & & \\
\hline $4364 *(1)$ & 0 & 1 & 1.0000 & & & & \\
\hline $4365 *(1)$ & 0 & 1 & 1.0000 & & & & $y$ \\
\hline
\end{tabular}


Table 2 Prevalence of virulence and resistance genes among S. aureus isolates

\begin{tabular}{|c|c|c|c|c|c|c|c|}
\hline $\begin{array}{l}\text { Virulence } \\
\text { genes }(\%)\end{array}$ & $\begin{array}{l}\mathrm{CM} \\
(\mathrm{n}=63)\end{array}$ & $\begin{array}{l}\text { BTM } \\
(\mathrm{n}=94)\end{array}$ & $P$-value & $\begin{array}{l}\text { Resistance } \\
\text { genes }(\%)\end{array}$ & $\begin{array}{l}\mathrm{CM} \\
(\mathrm{n}=63)\end{array}$ & $\begin{array}{l}\text { BTM } \\
(n=94)\end{array}$ & $P$-value \\
\hline aur (100) & 63 & 94 & 1.0000 & norA (99) & 62 & 94 & 0.4013 \\
\hline hla (100) & 63 & 94 & 1.0000 & blaZ (17) & 13 & 14 & 0.3501 \\
\hline$h l b(99)$ & 63 & 93 & 1.0000 & tetM (3) & 1 & 3 & 0.6495 \\
\hline$h \lg B(99)$ & 63 & 93 & 1.0000 & $d f r G(2)$ & 0 & 3 & 0.2746 \\
\hline$h \lg C$ (96) & 61 & 89 & 0.7027 & $\operatorname{ermB}(1)$ & 1 & 0 & 0.4013 \\
\hline$f i b(96)$ & 59 & 92 & 0.2196 & $\ln u A(1)$ & 0 & 1 & 1.0000 \\
\hline пис (95) & 61 & 88 & 0.4768 & $\ln u B(1)$ & 1 & 1 & 1.0000 \\
\hline icaD (94) & 61 & 87 & 0.3162 & mecA (1) & 1 & 0 & 0.4013 \\
\hline$h \lg A(94)$ & 58 & 89 & 0.5236 & tetK $(1)$ & 1 & 0 & 0.4013 \\
\hline splA (92) & 60 & 85 & 0.3638 & $\operatorname{vgaA}(1)$ & 0 & 1 & 1.0000 \\
\hline splB (92) & 60 & 85 & 0.3638 & & & & \\
\hline lukD (89) & 55 & 85 & 0.7296 & & & & \\
\hline $\operatorname{lukE}(81)$ & 49 & 78 & 0.4165 & & & & \\
\hline seu (69) & 36 & 72 & 0.0099 & & & & \\
\hline sem (68) & 34 & 72 & 0.0030 & & & & \\
\hline $\operatorname{sen}(68)$ & 36 & 70 & 0.0231 & & & & \\
\hline seo (66) & 33 & 71 & 0.0026 & & & & \\
\hline sei (66) & 34 & 69 & 0.0120 & & & & \\
\hline seg (45) & 23 & 47 & 0.0955 & & & & \\
\hline sple (16) & 15 & 10 & 0.0270 & & & & \\
\hline $\sec (5)$ & 2 & 5 & 0.7027 & & & & \\
\hline $\operatorname{sel}(5)$ & 2 & 5 & 0.7027 & & & & \\
\hline tst (5) & 2 & 5 & 0.7027 & & & & \\
\hline $\operatorname{scn}(3)$ & 3 & 1 & 0.3029 & & & & \\
\hline $\operatorname{seh}(3)$ & 4 & 0 & 0.0245 & & & & \\
\hline $\operatorname{seq}(2)$ & 3 & 0 & 0.0628 & & & & \\
\hline sak (2) & 3 & 0 & 0.0628 & & & & \\
\hline sek (2) & 3 & 0 & 0.0628 & & & & \\
\hline sea/sep (2) & 3 & 0 & 0.0628 & & & & \\
\hline
\end{tabular}


Table 3 Presence of SaPIs among S. aureus isolates

\begin{tabular}{|c|c|c|c|c|c|}
\hline MGE & $\begin{array}{l}\text { Strain/ } \\
\text { CC }\end{array}$ & $\begin{array}{l}\text { No. of isolates } \\
\text { (CM) }\end{array}$ & $\begin{array}{l}\text { No. of isolates } \\
\text { (BTM) }\end{array}$ & ST & Reference \\
\hline SaPIbov1 & RF122/151 & - & 1 & 705 & (Fitzgerald et al., 2001) \\
\hline SaPIbov1 & RF122/151 & 2 & 4 & 504 & (Fitzgerald et al., 2001) \\
\hline SaPIbov1 & $\mathrm{RF} 122 / 151$ & - & 1 & 71 & (Fitzgerald et al., 2001) \\
\hline SaPIbov2 & V329/126 & - & - & & (Cucarella et al., 2001) \\
\hline SaPIbov- $v \mathrm{Sa} \alpha$ & RF122/151 & - & 1 & 705 & (Herron-Olson et al., 2007) \\
\hline SaPIbov- $v \mathrm{Sa} \alpha$ & RF122/151 & 2 & 4 & 504 & (Herron-Olson et al., 2007) \\
\hline SaPIbov- $v \mathrm{Sa} \alpha$ & $\mathrm{RF} 122 / 151$ & - & 1 & 71 & (Herron-Olson et al., 2007) \\
\hline SaPIbov4 & BA4/97 & - & 1 & $4365^{*}$ & (Viana et al., 2010) \\
\hline SaPIbov4 & BA4/97 & - & 1 & $4363 *$ & (Viana et al., 2010) \\
\hline SaPIbov4 & BA4/97 & - & 1 & $3898^{*}$ & (Viana et al., 2010) \\
\hline SaPIbov4 & BA4/97 & 1 & - & $3896^{*}$ & (Viana et al., 2010) \\
\hline SaPIbov4 & BA4/97 & 1 & 7 & $3891 *$ & (Viana et al., 2010) \\
\hline SaPIbov4 & BA4/97 & & 1 & 706 & (Viana et al., 2010) \\
\hline SaPIbov4 & BA4/97 & 8 & 1 & 97 & (Viana et al., 2010) \\
\hline SaPIbov4 & BA4/97 & 2 & 6 & 71 & (Viana et al., 2010) \\
\hline SaPIbov4 & BA4/97 & 6 & 1 & 50 & (Viana et al., 2010) \\
\hline SaPIbov5 & JP5338/- & - & 1 & $4365^{*}$ & (Viana et al., 2010) \\
\hline SaPIbov5 & JP5338/- & - & 1 & $4363^{*}$ & (Viana et al., 2010) \\
\hline SaPIbov5 & JP5338/- & - & 1 & $3898^{*}$ & (Viana et al., 2010) \\
\hline SaPIbov5 & JP5338/- & 1 & - & $3896 *$ & (Viana et al., 2010) \\
\hline SaPIbov5 & JP5338/- & 1 & 6 & $3891 *$ & (Viana et al., 2010) \\
\hline SaPIbov5 & JP5338/- & 2 & 6 & 71 & (Viana et al., 2010) \\
\hline SaPIbov5 & JP5338/- & 6 & 1 & 50 & (Viana et al., 2010) \\
\hline$v$ SaBov & RF122/151 & & 2 & $3899 *$ & (Herron-Olson et al., 2007) \\
\hline$v$ SaBov & $\mathrm{RF} 122 / 151$ & - & 2 & $3900 *$ & (Herron-Olson et al., 2007) \\
\hline$v \mathrm{SaBov}$ & RF122/151 & 1 & 2 & 705 & (Herron-Olson et al., 2007) \\
\hline$v$ SaBov & RF122/151 & 2 & 4 & 504 & (Herron-Olson et al., 2007) \\
\hline$v$ SaBov & $\mathrm{RF} 122 / 151$ & 11 & 19 & 151 & (Herron-Olson et al., 2007) \\
\hline$v$ SaBov & RF122/151 & - & 1 & 7 & (Herron-Olson et al., 2007) \\
\hline$\varphi 12 \mathrm{bov}$ & RF122/151 & - & 2 & $3899 *$ & (Herron-Olson et al., 2007) \\
\hline$\varphi 12 \mathrm{bov}$ & RF122/151 & - & 2 & $3900^{*}$ & (Herron-Olson et al., 2007) \\
\hline$\varphi 12$ bov & $\mathrm{RF} 122 / 151$ & 1 & 2 & 705 & (Herron-Olson et al., 2007) \\
\hline$\varphi 12 \mathrm{bov}$ & $\mathrm{RF} 122 / 151$ & 2 & 4 & 504 & (Herron-Olson et al., 2007) \\
\hline$\varphi 12 \mathrm{bov}$ & RF122/151 & 11 & 17 & 151 & (Herron-Olson et al., 2007) \\
\hline$\varphi \mathrm{SaBov}-v$-Sa $\beta \varphi$ & RF122/151 & - & 2 & $3899^{*}$ & (Herron-Olson et al., 2007) \\
\hline$\varphi$ SaBov- $v$-Sa $\beta \varphi$ & RF122/151 & - & 1 & $3900 *$ & (Herron-Olson et al., 2007) \\
\hline$\varphi \mathrm{SaBov}-v$-Sa $\beta \varphi$ & RF122/151 & 1 & 1 & 705 & (Herron-Olson et al., 2007) \\
\hline$\varphi \mathrm{SaBov}-v$-Sa $\beta \varphi$ & RF122/151 & 2 & 3 & 504 & (Herron-Olson et al., 2007) \\
\hline$\varphi \mathrm{SaBov}-v$-Sa $\beta \varphi$ & RF122/151 & 6 & 15 & 151 & (Herron-Olson et al., 2007) \\
\hline
\end{tabular}

The table shows seven S. aureus pathogenicity islands (SaPIs) identified among 94 isolates from bulk tank milk (BTM) and 63 isolates

from clinical mastitis (CM) and the STs for these isolates are shown. SaPI bov2 was not identified in any of the isolates. The number of

open reading frames (ORFs) associated with each of the SaPIs are shown together with the strains and their CCs, from where the SaPIs

were initially found. References for each SaPI are shown and new STs are marked with an asterisk (*). Unk: Unknown 